\title{
ESTUDO SOBRE TRIATOMÍNEOS E RESERVATÓRIOS SILVESTRES DE TRYPANOSOMA CRUZI NO ESTADO DO PARANÁ, SUL DO BRASIL. RESULTADOS PRELIMINARES
}

\author{
Max Jean de Ornelas Toledo, João Balduino Kühl, Sandra Vieira da Silva, \\ Marcos Venício de Gasperi e Silvana Marques de Araújo
}

\begin{abstract}
Em localidades da zona rural de cinco municípios da região noroeste do Paraná adultos e ninfas de Triatoma sordida foram capturados em 21 (41,2\%) de 51 unidades domiciliares pesquisadas. Foram capturados 154 exemplares de T. sordida e 2 Panstrongylus megistus. De 135 exemplares examinados 58 (43,0\%) apresentavam o Trypanosoma tipo cruzi. Constatou-se também a infecção em 57,1\% (4/7) dos gambás (Didelphis sp) examinados. O peridomicílio apresentou-se mais infestado que o intradomicílio, sendo a casa de madeira abandonada a construção mais freqüente (34,7\% do total investigado) e com maior taxa de infestação (53,9\%). Os dados mostram um elevado indice de infestação da zona rural por espécies secundárias de triatomineos com altas taxas de infecção por flagelados do tipo T. cruzi, em uma área endêmica para a doença de Chagas já em fase de vigilância epidemiológica.
\end{abstract}

Palavras-chaves: Doença de Chagas. Vetores. Reservatórios silvestres. Área endêmica. Paraná.

No sul do Brasil, a infecção humana por Trypanosoma cruzi é comprovadamente endêmica nos Estados do Rio Grande do Sul e do Paraná. O Inquérito Sorológico Nacional para doença de Chagas (1975-1980)5 estimou uma prevalência de $4 \%$ para o Paraná, o que proporciona um número esperado de 166.511 positivos para uma população de 4.164 .943 habitantes da área rural e coloca este estado em quarto lugar no Brasil quanto ao número estimado de pacientes chagásicos. Com estes números, o Estado do Paraná fica atrás apenas de Minas Gerais, Bahia e Rio Grande do Sul15. Além deste dado, os escassos registros sobre a doença neste estado, publicados nas décadas de 40 e 50, restrigem-se a documentar a presença de vetores infectados e domiciliados e de casos humanos através da sorologia convencional1 1213 16. Estes autores registraram o primeiro encontro de Panstrongylus megistus naturalmente infectado com T. cruzi em Jataí, município situado às margens do rio Tibagi ao

Setor de Parasitologia Básica, Departamento de Análises Clínicas da Universidade Estadual de Maringá

Apoio Financeiro: Fundação Nacional de Saúde (FNS)/PIBIC. Endereço para correspondência: Dr. Max Jean de Ornelas Toledo. Departamento de Análises Clínicas, Universidade Estadual de Maringá. Caixa Postal 331, 87020-900, Maringá, PA. Fax (044) 223-2627.

Recebido para publicacão em 24/06/96. norte do estado, em 1917. Nesta época, foi encontrado também o Triatoma sordida em Foz do Iguaçu. Já o primeiro registro da presença do Triatoma infestans, também naturalmente infectado, só veio a ocorrer em 1941 no município de Piraquara. A maioria destes triatomíneos foi encontrada em municípios do norte próximos à divisa com o Estado de São Paulo, para onde ocorreu uma intensa migração, principalmente de mineiros, na primeira metade deste século. $\mathrm{Na}$ década de 70 , foi observada uma prevalência global de 7,4\% entre doadores em bancos de sangue de Londrina, norte do Estado3.

Nos últimos anos, o Departamento de Análises Clínicas da Universidade Estadual de Maringá detectou uma taxa de $6,3 \%$ de indivíduos com sorologia positiva para T. cruzi, residentes em vários municípios da região noroeste do Estado10 11. O encontro desta taxa de indivíduos sorologicamente positivos nesta região é um dado importante pois, embora seja considerada área de vigilância epidemiológica a ex-SUCAN em investigações nos anos de 1980 e 1989 capturou $P$. megistus e T. sordida no intra e peridomicílio de diferentes municípios desta região.

No presente trabalho, apresentamos o resultado preliminar de capturas de triatomíneos e reservatórios silvestres da doença de Chagas, realizadas durante o mês de agosto de 1995 na 
Toledo MJO, Kühl JB, Silva SV, Gasperi MV, Araújo SM. Estudo sobre triatomíneos e reservatórios silvestres de Trypanosoma cruzi no Estado do Paraná, sul do Brasil. Resultados preliminares. Revista da Sociedade Brasileira de Medicina Tropical 30:197-203, mai-jun, 1997.

zona rural de cinco municípios do noroeste do Estado do Paraná, relatando a distribuição e a prevalência das espécies de triatomíneos. Foi também determinada a taxa de infecção por protozoários tipo $T$. cruzi de triatomíneos e reservatórios capturados.

\section{MATERIAL E MÉTODOS}

Área de abrangência. A área de abrangência deste trabalho envolve 24 localidades da zona rural de cinco municípios (Maringá, Sarandí, Paiçandú, Dr. Camargo e Floresta) da Mēsorregião Noroeste Paranaense (Figura 1). Estes municípios

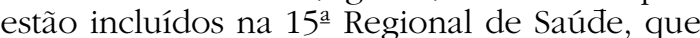
é composta por um total de 29 municípios, onde vivem 548.763 habitantes. O clima é subtropical úmido com preciptações médias anuais entre $1250 \mathrm{~mm}$ e $1500 \mathrm{~mm}$ e a vegetação primária foi extensivamente devastada. No entanto, ainda existem reservas de vegetação original da floresta subtropical latifoliada subcaducifólia no interior do Brasil. Os solos são intensamente explorados para cultivo de soja, trigo e milho dentre outros produtos.

Captura dos triatomíneos. Os levantamentos de triatomíneos foram realizados durante quatro dias do mês de agosto com o apoio da Fundação Nacional de Saúde (FNS), Distrito Sanitário de Londrina, Subdistrito de Maringá, nas localidades com "denúncias de focos" realizadas pelos moradores. Estas localidades são constituídas por unidades domiciliares (UD), variando de uma a sete UD/localidade. Considera-se como UD a casa sede, geralmente habitada e os anexos 15 , acrescida de uma ou mais casas de madeira desabitadas, via de regra presentes nas proximidades e que foram consideradas também como anexo. A equipe vasculhava casas habitadas, casas abandonadas e peridomicílio com os respectivos anexos e submetia um dos moradores da casa a uma ficha de investigação epidemiológica. Esta ficha foi elaborada segundo modelo apresentado no manual de normas técnicas para inquérito sorológico para avaliação do Programa de Controle da Doença de Chagas (PCDCH)9. A análise destas fichas nos forneceu a taxa de infestação das unidades domiciliares assim como outras informações que serão apresentadas.

Exame dos triatomíneos. Os triatomíneos capturados foram encaminhados ao Laboratório de doença de Chagas do Setor de Parasitologia Básica da Universidade Estadual de Maringá
(UEM) para identificação específica, determinação da taxa de infecção e isolamento de amostras de protozoários flagelados que serão utilizadas em estudos posteriores. Parte dos triatomíneos foi examinada por compressão abdominal e se necessário processava-se a dissecação do mesmo em solução salina. Este material foi examinado ao microscópio pesquisando-se protozoários flagelados. Quando positivo, as fezes e/ou conteúdo intestinal do triatomíneo eram inoculados em camundongos albinos machos com 3-6 semanas de idade. A cultura do conteúdo intestinal do triatomíneo em meio LIT - Liver Infusion Tryptose foi realizada para outro lote de triatomíneos como sugerido por Bronfen e cols 4 . Os insetos foram imersos em solução esterelizante de White e, posteriormente o conteúdo intestinal destes era retirado em condições assépticas e semeado em tubos contendo $5 \mathrm{ml}$ de meio LIT acrescido de $6 \mathrm{mg} / \mathrm{ml}$ de amplacilina. Os tubos eram mantidos a $28^{\circ} \mathrm{C}$ e as culturas eram examinadas a fresco 15 dias após sua execução.

Captura dos mamíferos silvestres. Alguns mamíferos considerados como reservatórios da doença de Chagas foram capturados no peridomicílio das localidades; foram também examinados mamíferos procedentes do Parque do Ingá, localizado em área urbana da cidade de Maringá (PR), onde um minizoológico recebe ocasionalmente animais silvestres capturados por moradores da região. Estes animais se encontravam em seus ninhos no momento da captura, à qual era realizada manualmente. Estes ninhos eram encontrados no forro das casas de madeira podendo estar ou não infestados por triatomíneos.

Exame dos mamíferos silvestres. Os animais silvestres, 7 gambás (Didelphis sp), 1 camundongo (Mus musculus) e 1 cutia (Dasyprocta ssp) foram submetidos à hemocultura em meio LIT e à cultura de fragmentos de fígado, baço e medula óssea em meio Blood Agar Base (DIFCO). Os animais foram imobilizados e anestesiados com éter e o sangue para hemocultura era obtido assepticamente, por punção intracardíaca para os gambás e cutia e por punção retroorbital para o camundongo. Cerca de $0,5 \mathrm{ml}$ do sangue de cada animal era distribuído em 2 tubos contendo $5 \mathrm{ml}$ de meio LIT. Os fragmentos da medula óssea foram obtidos por punção femural e, assim como aqueles do fígado e do baço, foram semeados em 4 tubos contendo cada um $4 \mathrm{ml}$ do meio Blood Agar Base. Todo o material era 
Toledo MJO, Kühl JB, Silva SV, Gasperi MV, Araújo SM. Estudo sobre triatomíneos e reservatórios silvestres de Trypanosoma cruzi no Estado do Paraná, sul do Brasil. Resultados preliminares. Revista da Sociedade Brasileira de Medicina Tropical 30:197-203, mai-jun, 1997.

incubado a $28^{\circ} \mathrm{C}$ e examinado 30,45 e 60 dias após. A taxa de infecção destes reservatórios foi determinada e amostras dos flagelados dos animais positivos, assim como aquelas obtidas de triatomíneos, foram isoladas, avaliadas em esfregaços corados pelo Giemsa e imediatamente criopreservadas em nitrogênio líquido.

\section{RESULTADOS}

A Tabela 1 mostra que apenas duas espécies de triatomíneos foram capturadas. O T. sordida correspondeu a 98,7\% (154 exemplares) do total de 156 exemplares capturados. Entre os exemplares desta espécie se encontravam tanto ninfas quanto adultos e os dois exemplares de $P$. megistus capturados eram ninfas. $O$ número de localidades visitadas e a taxa de infestação das habitações por triatomíneos nos 5 municípios investigados são apresentados na Tabela 2. Das 21 unidades domiciliares infestadas foram encontrados triatomíneos em apenas 2 casas habitadas no momento da visita. Em uma dessas casas foram encontrados adultos e ninfas e na outra apenas um exemplar adulto. Nas demais 19 unidades domiciliares, somente o peridomicílio apresentou colônias de triatomíneos. Todos os cinco municípios investigados apresentaram alta taxa de infestação das unidades domiciliares. O município de Paiçandú, onde foi investigado maior número de localidades (9), foi onde observou-se a taxa de infestação de $52,5 \%$ das unidades domiciliares (Tabela 2) e o maior número de exemplares, 78 do total de 156 capturados (Tabela 3). A espécie P. megistus só foi encontrada neste município (Figura $1 \mathrm{e}$ Tabela 3). A taxa de infestação atingiu 80\% no município de Dr. Camargo, porém um número pouco representativo (apenas 5) de domicílios foi investigado (Tabela 2).

Tabela 1 - Espécies de triatomíneos capturadas na zona rural de municípios do noroeste do Estado do Paraná, Brasil.

\begin{tabular}{lcc}
\multicolumn{4}{l}{ municipios do noroeste do Estado do Paraná, Brasil. } \\
\hline Espécie & № de exemplares & $\%$ \\
\hline T. sordida & 154 & 98,7 \\
$P$. megistus & 2 & 1,3 \\
\hline Total & 156 & 100,0
\end{tabular}

A casa com parede de madeira foi o domicílio mais freqüente, correspondendo a $80 \%$ dos domićlios investigados (Figura 3a). Não foram encontradas casas de adobe nem casas de pau-a-pique na região estudada. Os anexos domiciliares mais freqüentes foram a casa de madeira abandonada, correspondendo a 34,7\% dos anexos investigados e apresentando uma taxa de infestação por triatomíneos de 58,9\% (Figuras 2 e 3b). Em segundo lugar apareceu o paiol (21,2\%), sendo que $25,0 \%$ deles apresentaram-se infestado. Em seguida o chiqueiro e o galinheiro apareceram com a mesma freqüência (16,0\% dos anexos), embora o chiqueiro $(16,7 \%)$ mostrou-se mais infestado que o galinheiro $(8,3 \%)$ (Figura $3 b)$.

Tabela 2 - Taxa de infestação por triatomíneos em unidades domiciliares de diferentes localidades investigadas de municípios do noroeste do Estado do Paraná, Brasil.

\begin{tabular}{|c|c|c|c|c|}
\hline \multirow[b]{2}{*}{ Município } & \multirow{2}{*}{$\begin{array}{c}\text { № de } \\
\text { localidades }\end{array}$} & \multicolumn{2}{|c|}{ № de UD } & \multirow{2}{*}{$\begin{array}{c}\% \text { de } \\
\text { infestação }\end{array}$} \\
\hline & & investigadas & infestadas & \\
\hline Paiçandú & 9 & 19 & 10 & 52,5 \\
\hline Floresta & 7 & 7 & 2 & 28,6 \\
\hline Sarandi & 5 & 13 & 2 & 15,4 \\
\hline Dr. Camargo & 2 & 5 & 4 & 80,0 \\
\hline Maringá & 1 & 7 & 3 & 42,9 \\
\hline Total & 24 & 51 & 21 & 41,2 \\
\hline
\end{tabular}

UD = unidades domiciliares.

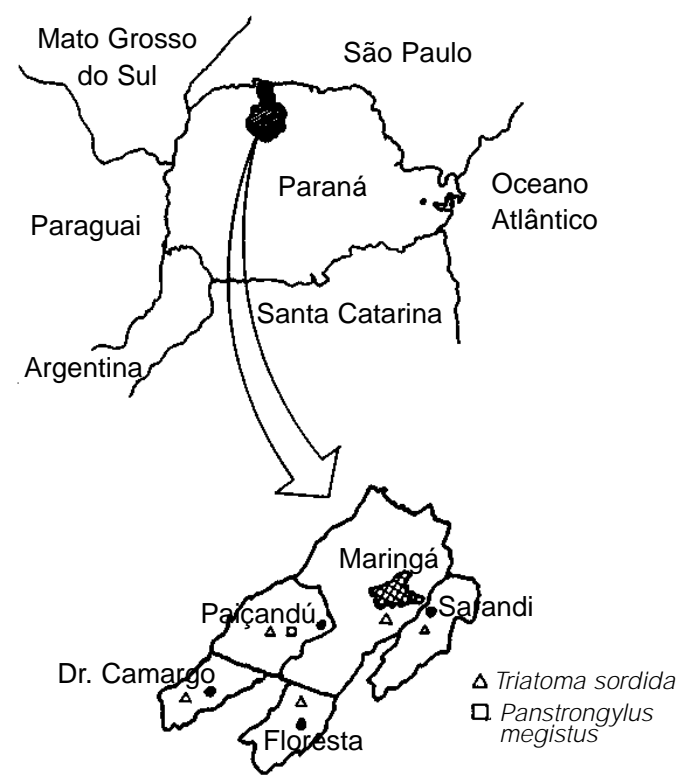

Figura 1 - Mapa do Estado do Paraná destacando a área de abrangência da $15^{-}$Regional de Saúde (área achuriada) onde se encontram os cinco municipios nos quais foram realizadas capturas de triatomíneos. Apresenta também a distribuição das espécies por município. Agosto/1995.

Do total de 56 moradores (média de 3,5 \pm 1,4 moradores por casa), $10(17,8 \%)$ relataram ter sido picados por "chupança" ou "bicho barbeiro", nomes mais comuns para designar os triatomíneos na região. Estes indivíduos relataram um tempo médio na residência atual de 12 anos. 
Toledo MJO, Kühl JB, Silva SV, Gasperi MV, Araújo SM. Estudo sobre triatomíneos e reservatórios silvestres de Trypanosoma cruzi no Estado do Paraná, sul do Brasil. Resultados preliminares. Revista da Sociedade Brasileira de Medicina Tropical 30:197-203, mai-jun, 1997.

Tabela 3 - Taxa de infeccão por Trypanosoma tipo cruzi segundo a espécie de triatomíneo e o município de captura, no noroeste do Estado do Paraná, Brasil.

\begin{tabular}{lccccc}
\hline & & \multicolumn{3}{c}{ o de exemplares } & \% de \\
\cline { 3 - 5 } Município & Espécie & capturados & examinados & positivos & infecção \\
\hline Paiçandú & T. sordida & 76 & 65 & 34 & 52,3 \\
& P. megistus & 2 & 2 & 1 & 50,0 \\
Floresta & T. sordida & 22 & 19 & 12 & 63,2 \\
Sarandi & T. sordida & 13 & 11 & 8 & 72,7 \\
Dr. Camargo & T. sordida & 22 & 19 & 2 & 10,5 \\
Maringá & T. sordida & 21 & 19 & 1 & 5,3 \\
\hline Total & & 156 & 135 & 58 & 43,0
\end{tabular}

A Tabela 3 mostra que de um total de 135 exemplares de triatomíneos examinados, 58 apresentaram-se positivos para flagelados o que corresponde a um índice de infecção geral de 43,0\%. Todos os cinco municípios investigados apresentaram elevadas taxas de infecção para os triatomíneos: a menor percentagem de infecção do vetor foi observada naqueles capturados na zona rural de Maringá onde apenas $5,3 \%$ estavam infectados e a maior taxa foi observada em Sarandí onde $72,7 \%$ dos insetos examinados apresentaram flagelados (Tabela 3).

a
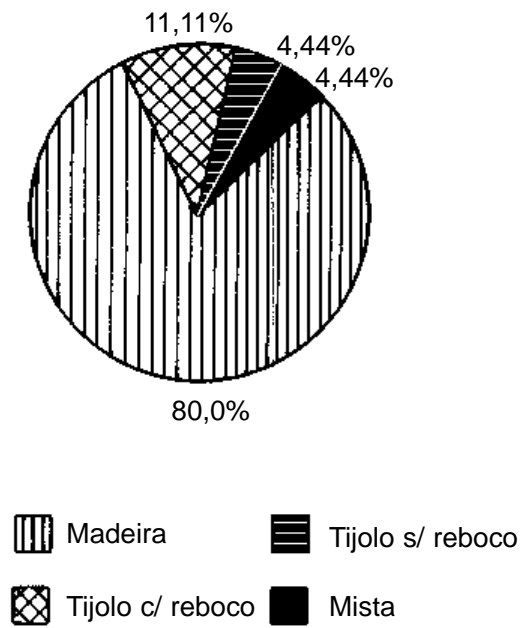

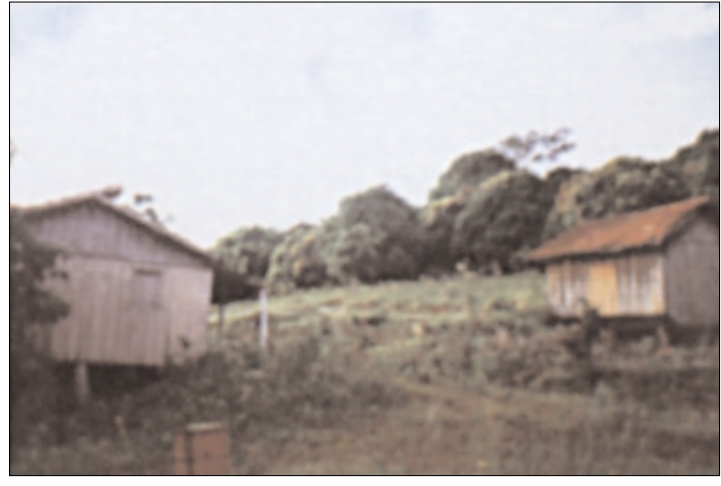

Figura 2 - Anexos domiciliares: casa de madeira com teto de telha desabitada servindo como depósito de cereais e madeira infestada por T. sordida $e \mathrm{P}$. megistus (à esquerda); paiol de madeira (à direita) na zona rural do município de Paiçandú, PR. Agosto/1995.

Dos 7 marsupiais e 2 roedores silvestres examinados, apenas o gambá (Didelphis sp.) foi encontrado naturalmente infectado (Tabela 4). Em quatro $(57,1 \%)$ de sete exemplares de marsupiais examinados comprovou-se, por hemocultura e cultura de medula óssea,

\section{b}

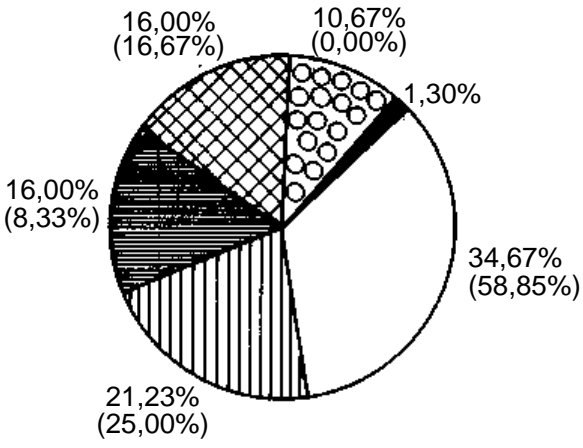

Casa mad. aband.

Galinheiro 8 Curral
$\$$ Chiqueiro $\square$ Outros

Figura 3 - a) Tipos de parede dos domicílios investigados para triatomíneos; b) Freqüência dos anexos domiciliares e (taxa de infestação por triatomineos), na zona rural do noroeste do Paraná. Agosto/1995. 
Toledo MJO, Kühl JB, Silva SV, Gasperi MV, Araújo SM. Estudo sobre triatomíneos e reservatórios silvestres de Trypanosoma cruzi no Estado do Paraná, sul do Brasil. Resultados preliminares. Revista da Sociedade Brasileira de Medicina Tropical 30:197-203, mai-jun, 1997.

a infecção por flagelados. Estes parasitas foram inoculados em camundonos albinos jovens infectando-os e mostraram-se morfologicamente indistinguíveis do T. cruzi. A partir dos 58 triatomíneos e dos 4 marsupiais infectados foram isoladas 49 amostras do parasita. Trinta e três amostras através de inoculação em camundongos e/ou recuperação por hemocultura e 16 através da xenocultura. Todas as amostras isoladas foram criopreservadas em nitrogênio líquido e estão sendo caracterizadas através de diferentes parâmetros biológicos tais como: infectividade, curva de parasitemia e mortalidade em camundongos, sensibilidade à quimioterápicos específicos, cinética de crescimento em meio LIT, tropismo tissular e alterações histopatológicas em camundongos.

\begin{tabular}{|c|c|c|c|c|}
\hline \multirow[b]{2}{*}{ Animal } & \multirow{2}{*}{$\begin{array}{l}\text { Localidade } \\
\text { da captura }\end{array}$} & \multicolumn{2}{|c|}{ Número de animais } & \multirow{2}{*}{$\begin{array}{c}\% \text { de } \\
\text { infecção }\end{array}$} \\
\hline & & capturados & infectados* & \\
\hline \multirow[t]{2}{*}{ Didelphis sp } & $\begin{array}{l}\text { zona rural de } \\
\text { Sarandi }\end{array}$ & 2 & 2 & 100,0 \\
\hline & $\begin{array}{l}\text { Parque do } \\
\text { Ingá, Maringá }\end{array}$ & 5 & 2 & 40,0 \\
\hline Dasyprocta sp & $\begin{array}{l}\text { Parque do } \\
\text { Ingá, Maringá }\end{array}$ & 1 & 0 & 0,0 \\
\hline Mus musculus & $\begin{array}{l}\text { zona rural de } \\
\text { Sarandí }\end{array}$ & 1 & 0 & 0,0 \\
\hline Total & & 9 & 4 & 44,4 \\
\hline
\end{tabular}
cultura de medula óssea em meio Blood Agar Base.

\section{DISCUSSÃO}

$\mathrm{Na}$ década de 40, segundo registro de pesquisadores, apenas as cafuas apresentavam colônias de "barbeiros" no Paraná. Nas casas de madeira, numerosas na zona rural deste estado até nos dias atuais, só foram encontrados basicamente exemplares adultos. Pensava-se então que esse tipo de habitação servia como criadouros do vetor apenas ocasionalmente13. A espécie predominantemente observada foi o T. infestans. Passaram-se mais de 50 anos e o quadro hoje observado é bem diferente, mesmo sendo nossos resultados preliminares e o número de triatomíneos e reservatórios capturados muito pequeno. Oitenta por cento das casas investigadas eram de parede de madeira com teto de telha. Destas, apenas uma casa habitada apresentou colônias de T. sordida no intradomicílio. Entretanto, $58,9 \%$ das casas desabitadas estavam infestadas por essa espécie de triatomíneo. A espécie $T$. sordida pertence aos domínios do cerrado onde é encontrada com mais freqüência em "paus sêcos", onde certamente se encontra sua fonte alimentar, mostrando nítida associação entre esta espécie e ecótopos de madeira6. Provavelmente, as casas de madeira desabitadas servem de abrigo a marsupiais ou outras fontes alimentares de triatomíneos das quais, praticamente eles nunca se afastam.

Neste trabalho foi observado que a espécie predominante atualmente nesta região é o $T$. sordida que correspondeu a 98,7\% dos exemplares capturados. Este dado informa sobre a eficácia do PCDCH nesta região do Paraná, uma vez que a espécie domiciliar $T$. infestans predominante nas décadas anteriores à implantação do programa não foi mais encontrada na investigação atual. Já o T. sordida é espécie freqüentemente encontrada no peridomicílio mas com capacidade invasiva em relação à casa15. A intervenção com inseticida seria indicada pelos elevados índices de captura de exemplares de $T$. sordida infectados no peridomicílio e que poderiam colonizar o intradomicílio a partir de uma densidade crítica, quando a oferta alimentar no peridomicílio fosse esgotada6 uma vez que os anexos infestados localizavam-se próximos às residências. Outra observação digna de nota é a presença de $P$. megistus infectado com flagelados, no peridomicílio, colonizando ecótopos artificiais no Paraná. Na investigação atual essa espécie foi capturada no peridomicílio embora ela tenha sido encontrada colonizando casas de madeira habitadas em outros municípios da região noroeste do Paraná (MJO Toledo: dados não publicados). Estes achados justificam a imediata asperção de inseticida embora o $P$. megistus na região sul do Brasil seja encontrado em ecótopos silvestres, invadindo apenas esporadicamente a casa e constituindo colônias no domicílio muito raramente15.

Um outro dado que chama a atenção foi a alta taxa de infecção geral por $T$. tipo cruzi $(43,0 \%)$ dos triatomíneos examinados contrariando a maioria dos registros na literatura2 7 14. Estes índices elevados de infecção podem ser em parte devido ao estreito convívio destes vetores com marsupiais. Como relatado, quatro $(57,1 \%)$ dos sete gambás examinados neste estudo apresentavam-se infectados. Além deste fato, boa parte dos triatomíneos foi capturada em ninhos de gambá localizados no forro das 
Toledo MJO, Kühl JB, Silva SV, Gasperi MV, Araújo SM. Estudo sobre triatomíneos e reservatórios silvestres de Trypanosoma cruzi no Estado do Paraná, sul do Brasil. Resultados preliminares. Revista da Sociedade Brasileira de Medicina Tropical 30:197-203, mai-jun, 1997.

casas de madeira abandonadas. A região meridional do território brasileiro é relatada como área de ocorrência de $P$. megistus associado a mamíferos sinantrópicos, representados principalmente por marsupiais $\mathrm{e}$ roedores. Na região estudada observamos a frequënte associação entre o T. sordida e estes animais, principalmente com os gambás o que aumenta a capacidade vetorial desta espécie, embora, segundo a literatura, ela apresente marcada ornitofilia em diferentes regiões do país 15 . Acrescente-se a isto o fato de que parece ser a densidade populacional deste reservatório elevada tanto na zona rural como nos parques da cidade de Maringá, em vista das informações da população e da bióloga responsável por estes parques.

Segundo Forattini e cols (1984) 8 a invasão e colonização de ecótopos artificiais, os índices de infecção natural elevados e repastos ocasionais em sangue humano, são fatores indicativos da ocorrência de transmissão. Seja no domicílio ou em seus anexos, a convivência com triatomíneos e mamíferos infectados aumenta a possiblidade da infecção humana. Estes dados são preocupantes já que estão sendo confirmados em um momento em que o país se prepara para considerar a doença de Chagas controlada, de acordo com as discussões ocorridas em novembro de 1995 na XI Reunião Anual sobre Pesquisa Aplicada em Doença de Chagas, Uberaba, MG.

$\mathrm{Na}$ continuação destes estudos, o próximo passo seria determinar a fonte alimentar destes triatomíneos e o potencial de transmissão do T. cruzi pelo T. sordida. Além de capturar um maior número de triatomíneos, estendendo essa investigação para outras regiões do estado.

\section{SUMMARY}

In the rural area of five municipalities of the Northeast of the State of Paraná, Brazil, 154 adults or nymphs of Triatoma sordida and 2 Panstrongylus megistus were captured in 21 (41.2\%) of 51 dwellings at the peridomestic sites. Trypanosoma cruzi-like organisms were found in 58 out of the 135 (43.0\%) triatomines. Moreover, it was also found in the blood of 4/7 opossum (Didelphis sp). The triatomine infestation was more prevalent in the peridomicile than inside house. The forsaken wooden house presented the highest level of infestation (53.8\%). Although the rural area of the Northeast of the State of Parana is considered under epidemiologic surveillance it still presents a high level of peridomestic infestation by triatomines highly infected with T. cruzi-like organisms.

Key-words: Chagas' disease. Vectors. Silvatic reservoirs. Paraná.

\section{AGRADECIMENTOS}

Agradecemos a ajuda dos técnicos da FNS, Élcio Silvestre dos Santos, Demilson Rodrigues dos Santos e Cícero Miranda da Silva, que com experiência e presteza foram fundamentais para a realização deste trabalho; o auxílio técnico de Ângela Cristina Benedito e à Professora Thaís Gomes Verzignassi Silveira pela pesquisa de flagelados nas vísceras de animais silvestres.

\section{REFERÊNCIAS BIBLIOGRÁFICAS}

1. Almeida AS. Doença de Chagas no Norte do Paraná. Anais Paulistas de Medicina e Cirurgia 56:29-36,1948.

2. Barretto MP. Epidemiologia. In: Brener Z,Andrade Z. Trypanosoma cruzi e Doença de Chagas. $1^{\mathrm{a}}$ edição, Guanabara Koogan, Rio de Janeiro p.89151, 1979.

3. Baldy JLS,Takaoka L, Pereira JD, Calixto AA, Duarte EL. Prevalência da infecção por Trypanosoma cruzi, em 1975, em dois bancos de sangue de Londrina, Paraná, Brasil. Revista de Saúde Pública de São Paulo 12: 409-416, 1978.

4. Bronfen E, Rocha FSA, Machado GBN, Perilon MM, Romanha AJ, Chiari E. Isolamento de amostras do Trypanosoma cruzi por xenodiagnóstico e hemocultura de pacientes na fase crônica da doença de Chagas. Memórias do Instituto Oswaldo Cruz 84:237-240, 1989.

5. Camargo ME, Silva GR, Castilho EA, Silveira AC. Inquérito sorológico da prevalência de infecção chagásica no Brasil, 1975-1980. Revista do Instituto de Medicina Tropical de São Paulo 26:192-204, 1984.

6. Diotauiti L. Importância atual e perspectivas de controle de Triatoma sordida em Minas Gerais. Tese de doutorado, Universidade Federal de Minas Gerais, Belo Horizonte, MG, 1991.

7. Diotaiuti L, Paula OR, Falcão PL, Dias JCP. Avaliação do programa de controle vetorial da doença de Chagas em Minas Gerais, Brasil, com referência especial ao Triatoma sordida. Boletin de la Oficina Sanitária 118: 211-219, 1995.

8. Forattini OP, Rabello EX, Ferreira OA, Rocha e Silva EO, Santos JFL. Aspectos ecológicos da 
Toledo MJO, Kühl JB, Silva SV, Gasperi MV, Araújo SM. Estudo sobre triatomíneos e reservatórios silvestres de Trypanosoma cruzi no Estado do Paraná, sul do Brasil. Resultados preliminares. Revista da Sociedade Brasileira de Medicina Tropical 30:197-203, mai-jun, 1997.

trypanossomíase americana. XXI - Comportamento de espécies de triatomíneos na reinfestação do intra e peridomicílio. Revista de Saúde Pública de São Paulo 18: 185-208, 1984.

9. Fundação Nacional de Saúde. Manual de Normas Técnicas para Inquérito Sorológico para Avaliação do Programa de Controle da Doença de Chagas. Ministério da Saúde, Brasília, 1994.

10. Gomes ML, Bertolini DA, Silveira TGV, Lonardoni MCV, Arraes SMA. Investigação sorológica da doença de Chagas e isolamento do Trypanosoma cruzi em indivíduos de cinco municípios da região noroeste do Paraná. Revista da Sociedade Brasileira de Medicina Tropical 25 (supl III):94, 1992.

11. Gomes ML, Ramos M, Silveira TGV, Dias MLGG, Araújo SM. Isolation and biological characterization of Trypanosoma cruzi strains in North of Paraná, Brazil. Memórias do Instituto Oswaldo Cruz 85 (supl I):16, 1990.

12. Lôbo AGS, Borba AM, Souza J. Contribuição ao conhecimento da distribuição geográfica dos triatomíneos domiciliários e seus índices de infecção natural pelo Schizotrypanum cruzi no Estado do Paraná. Revista Brasileira de Malariologia e Doenças Tropicais, 571-584, 1953.

13. Simões AJP. Doença de Chagas no Estado do Paraná, Brasil. Esboço Epidemiológico. Memórias do Instituto Oswaldo Cruz 39:279-290, 1943.

14. Rodrigues VLCC, Ferraz Filho AN, Silva EOR, Lima VLC. Prevalência, índices de infecção e hábitos alimentares de triatomíneos capturados em uma área de vigilância epidemiológica. Revista da Sociedade Brasileira de Medicina Tropical 25:183190, 1992.

15. Silveira AC, Resende DF. Epidemiologia e controle da transmissão vetorial da doença de Chagas no Brasil. Revista da Sociedade Brasileira de Medicina Tropical 27 (supl. III): 11-22, 1994.

16. Souza-Araújo HC.A doença de Chagas no Paraná. Memórias do Instituto Oswaldo Cruz 52:477-485, 1954. 